\title{
A third of recommendations in Scottish guidelines are based on poorest evidence, finds study
}

This Research News story (BMJ 2014;348:g1428, doi:10.1136/ bmj.g1428)) incorrectly states that the SIGN guidelines on diabetes used only level D evidence for its recommendations.

In fact, the study found that the diabetes guidelines used lower levels of D evidence compared with other large guidelines, which showed a trend to use larger amounts of level D evidence.

Cite this as: BMJ 2014;348:g1474

๑ BMJ Publishing Group Ltd 2014 